\title{
A new type of photoionized code required for the new era of X-ray spectroscopy
}

\author{
S. Collin ${ }^{1}$, A.-M. Dumont ${ }^{1}$, and O. Godet ${ }^{2}$ \\ ${ }^{1}$ LUTH, Observatoire de Paris, Section de Meudon, 92195 Meudon Cedex, France \\ 2 CESR, 9 Av. du Colonel Roche, 31028 Toulouse Cedex 4, France
}

Received 12 January 2004 / Accepted 23 February 2004

\begin{abstract}
With the advent of the present and future spatial X-ray missions, it becomes crucial to model correctly the line spectrum of X-ray emitting media such as the photoionized plasma observed in the central regions of Active Galactic Nuclei (AGN), or in X-ray binaries. We have built a photoionization code, Titan, solving the transfer of a thousand lines and of the continuum with the "Accelerated Lambda Iteration" method, which is one of the most efficient and at the same time the most reliable for line transfer. In all other photoionization codes the line intensities are computed with the so-called "escape probability formalism", used in its simplest approximation. In a previous paper (Dumont et al. 2003), it was shown that this approximation leads to a wrong estimation of the emitted X-ray line intensities, especially in the soft X-ray range. The errors can exceed one order of magnitude in the case of thick media (Thomson thickness of the order of unity). In the present paper, we show that it also happens, but for different reasons, in the case of moderately thin media (Thomson thickness of 0.001 to 0.1), characteristic of the Warm Absorber in Seyfert 1 or of the X-ray emitting medium in Seyfert 2. Typically, the errors on the line fluxes and line ratios are of the order of $30 \%$ for a column density of $10^{20} \mathrm{~cm}^{-2}$, and a factor five for a column density of $10^{23} \mathrm{~cm}^{-2}$, in conditions giving rise to the spectra observed in these objects. We explain why this problem is less acute in cooler media, like the Broad Line Region of AGN. We show some examples of X-ray spectra appropriate for Seyfert 2 and for the Warm Absorber of Seyfert 1. We conclude that though it is quite important to introduce numerous accurate X-ray data in photoionization codes, it should be accompanied by more elaborate methods than escape probability approximations to solve the line transfer.
\end{abstract}

Key words. line: formation - radiative transfer - galaxies: seyfert - X-rays: general

\section{Introduction}

Since more than three decades, photoionization codes have been developed to compute the structure and the spectrum of photoionized media, such as HII regions, planetary nebulae, supernova remnants, envelopes of novae, Narrow Line Regions of Active Galactic Nuclei (AGN), etc. At the end of the seventies, these codes have been extended to denser and thicker media, like the Broad Emission Line Region (BLR) in quasars and AGN or the emission regions of X-ray binaries and cataclysmic variables. For this purpose, the formalism of "escape probability" has been introduced to take into account selfabsorption in lines while avoiding to solve the line transfer (Netzer 1975), and a new type of photoionization code has begun to be built. These codes - Cloudy (Ferland et al. 1988), XSTAR (Kallman \& Krolik 1995), ION (Netzer 1993), for instance - have now reached a high degree of sophistication, including very accurate atomic data, a large number of ions and transitions, and all the necessary processes allowing to use them in various physical conditions.

Send offprint requests to: S. Collin, e-mail: suzy . collin@obspm. fr
With the advent of the X-ray missions Chandra and XMM Newton, splendid spectra of various types of objects have been obtained in the soft X-ray range, showing tens of emission lines which can be used as diagnostics of the physical state of the emitting region. The best examples are Seyfert 2 galaxies, which display a rich X-ray line spectrum, most probably produced by the external part of the "Warm Absorber" of Seyfert 1, photoionized by the intense central continuum and seen in emission because the central continuum is hidden from our view (Antonucci \& Miller 1985). Typical column densities of this medium are $10^{21-23} \mathrm{~cm}^{-2}$ (Sako et al. 2000; Kinkhabwala et al. 2002; Ogle et al. 2003).

Most naturally, the photoionized codes developed for the BLR have been used to model the X-ray emitting regions of different objects, like the atmosphere of cataclysmic variables, $\mathrm{X}$-ray binaries, etc. These codes are also used to model the absorption lines observed in the UV and X-ray range in quasars and AGN. Numerous new X-ray atomic data have been introduced in the codes, in order to obtain the best possible accuracy on the X-ray spectrum. But the "escape probability approximation" still lies at the center of the computation of the line intensities. 
This formalism, developed in the sixties and in the seventies, can be very useful to perform rapid approximate computations, but it does not lead to a correct estimation of the source function, especially in the case of strongly interlocked transitions, including continuum ones. Moreover it uses as a local quantity a global one, computed by an integration over the whole medium; this is a dramatic extrapolation for very inhomogeneous media like photoionized plasma. These aspects have been completely overlooked during the last twenty years, but they become now crucial in the context of X-ray emitting media.

In a previous paper, Dumont et al. (2003, referred as D03) have shown that escape probability approximations, at least as they are used in the present codes, are unable to compute correct line intensities, within factors of ten, when the Thomson thickness of the medium is of the order of a few units, typical for the irradiated atmospheres of accretion discs in AGN and $\mathrm{X}$-ray binaries. In the present paper, we extend this study to less optically thick media, and we show that the intensities of emission lines are also not accurately computed for parameters typical of the Warm Absorber of Seyfert 1 galaxies and of the region giving rise to the $\mathrm{X}$-ray spectrum in Seyfert 2 galaxies ${ }^{1}$.

In the next section, we recall very briefly the essence of the problem, and we give a few examples in Sect. 3.

\section{Real transfer versus escape probability approximation}

\subsection{Failures of the escape probability approximation}

The escape probability method consists in decoupling the statistical equations of the levels giving rise to a line photon, from the transfer equation. This is performed by using in both equations a frequency integrated line profile which is identified with the probability of the photon to escape in a single flight from the medium. The problem has been amply treated since the sixties (cf. for instance "Radiation transport in spectral lines", by Athay 1972; and several reviews in "Methods of Radiative Transfer", Kalkoven 1984; "Numerical Radiative Transfer", Kalkoven 1987; Hubeny 2001...). There are different ways to use these escape probabilities, some being actually as sophisticated and time consuming as real transfer.

In photoionization codes, the emphasis is put on the atomic physics, to account for as many spectral features as possible, and to get a correct thermal and ionization equilibrium. The escape probability is treated in a "first order approximation", which amounts to replace in the population equations the net

\footnotetext{
${ }^{1}$ One should note that modeling an absorption spectrum is generally easier than an emission one. It requires only a correct computation of the thermal and ionization equilibrium, which give the fractional ion abundances, i.e. the populations of the ground levels, and thus the equivalent widths of the resonance lines, which can be compared to those deduced from the observations through a curve of growth analysis implying no line transfer. However it can happen (and this is indeed the case for the Warm Absorber), that emission lines are also produced by the absorbing medium, and one should thus worry about the line transfer.
}

radiative rate of excitation between two levels (called the Net Radiative Bracket, or NRB) by $n_{\mathrm{u}} A_{\mathrm{ul}} \rho_{\mathrm{ul}}$, where

$\rho_{\mathrm{ul}}=\frac{\left\{n_{\mathrm{u}}\left(A_{\mathrm{ul}}+B_{\mathrm{ul}} \int J_{\nu} \psi_{\nu} \mathrm{d} v\right)-n_{\mathrm{l}}\left(B_{\mathrm{lu}} \int J_{\nu} \phi_{\nu} \mathrm{d} v\right)\right\}}{n_{\mathrm{u}} A_{\mathrm{ul}}}$

is identified with the escape probability $\left(A_{\mathrm{ul}}, B_{\mathrm{ul}}\right.$ and $B_{\mathrm{lu}}$ are the Einstein excitation and deexcitation coefficients between the upper (u) and lower (l) levels of the transition, and $n_{\mathrm{u}}$ and $n_{1}$ are the number densities of the upper and lower levels). The escape probability intervenes also in the ionization and thermal equilibria, through the reabsorption of line photons, and in the computation of the line fluxes emerging from both sides.

The escape probability is a global quantity, expressed as a function of the optical thickness at the line center, $\tau_{0}$, between the emission point and the surface, but it depends through the damping constant (cf. the Appendix) also on physical parameters which vary in the medium, such as the temperature and the density. The local conditions are thus assumed to be valid all along the photon path. The escape probability is computed by an integration over the whole medium, considered as being homogeneous, which is far from being the case in photoionized plasma except when they are very thin. On the other hand, this global quantity is used in the determination of local rates, such as ionizations by line photons.

The problem is complicated when destruction processes occur before the escape of the photon (on the spot or along the photon path). This is the case if the medium is optically thick in the continuum underlying the line photon. Various approximate formulae are used to take continuum absorption into account in the escape probabilities. Note that in hot media, Comptonization acts as an absorption-diffusion process which should also be taken into account in the line transfer.

As shown in D03, no escape approximation can give accurate results when the Thomson thickness is of the order of unity or larger. Indeed local reabsorption of line photons by continuum processes implies a delicate balance between excitations of X-ray transitions by the intense underlying diffuse $\mathrm{X}$-ray continuum and the net rate of excitations by the diffuse line flux. It is not taken properly into account in escape probability approximations and it creates large differences in the emerging line spectrum.

When the medium is only moderately thick (Thomson thickness from 0.001 to 0.1 ), these processes are less important, but still the escape probabilities lead to inexact results, then for other reasons. For optically thick transitions, escape probabilities describe correctly the behavior of the source function deeply in the medium but not at the surface. These lines are formed close to the surface where their upper level populations are not computed correctly. This can be dramatic if the transition is strongly interlocked with other levels in a "multi-level" description. This is the case for the high resonance lines and the Balmer lines in hydrogen-like ions, or for the resonance lines and the intercombination and forbidden lines for helium-like ions. Avrett \& Loeser (1987) have shown that, unless methods coupling transfer and escape probabilities are used, large errors follow on the relative intensities of the lines even for a threelevel atom. 
Another problem rises for moderately thick or thin media. Whereas in a thick medium, the incident continuum is absorbed completely in resonance transitions and it does not play any role in line excitation (at least for the most intense lines), in the case of thin or moderately thick media as those considered in the present paper, the incident continuum can contribute appreciably to the line excitation. Indeed it is absorbed only at the line center, and a fraction is left in the wings. Its contribution should be added to the population equations, as $\rho_{\mathrm{ul}}$ includes only the diffuse radiation, which in this case is weak and does not contribute much to the excitations. The attenuation factor of the incident continuum is of the order of twice the escape probability towards the illuminated side (as it is defined in the Appendix, cf. Elitzur 1982), but it is again an approximation. When the medium is extremely thin, this factor is equal to unity, and the computation is exact.

Moreover, there is also the problem of frequency redistribution inside the lines. Resonance lines are broadened mainly by radiation damping, so the emission process is coherent in the frame of the atom. It is well-known since a long time that in this case the assumption of complete redistribution in a Voigt profile leads to strongly overestimate the line intensity (cf. for instance Milkey \& Mihalas 1973; Vernazza et al. 1981, who studied $\mathrm{L} \alpha$ in the solar spectrum), and a way to account better for partial redistribution (PRD) is to assume complete redistribution in the Doppler core. XSTAR assumes complete redistribution in a Doppler core for all lines, whereas in Cloudy, strong resonance lines are treated with partial redistribution within a Voigt profile, which amounts using for the escape probability an expression computed by Hummer \& Kunasz (1980) $P_{\text {esc }}\left(\tau_{0}\right)=\left[1+b\left(\tau_{0}\right) \tau_{0}\right]^{-1}$, where $b$ of the order of a few units. Note that this function is given only for a ratio of the continuum to line opacity smaller than $10^{-6}$, and it should be extrapolated for larger values, a common case in the present computations. Both treatments are close to complete redistribution in the Doppler core, and similar to the one used here for the resonance lines in our escape treatment (cf. the Appendix).

In summary, the escape probability method is strongly approximated, both in very thick and in moderately thick media, for different reasons.

One may therefore ask why it is possible to use this method to compute the optical-UV spectrum of the BLR, and not the $\mathrm{X}$-ray spectrum of a photoionized medium.

Consider first an X-ray emitting medium photoionized by an intense X-ray continuum, i.e. with a large "ionization parameter", defined as the radiation flux to the gas density ratio. The heavy elements are highly ionized. If the medium is only moderately thick, the continuum underlying the $\mathrm{X}$-ray lines can nevertheless be optically thick, owing to the presence of several ion species in important proportions at a given point in the medium (we will illustrate this discussion later in showing which ions are important for absorbing the continuum at the position of the OVIII L $\alpha$ line).

Consider now a less ionized medium like the BLR. The ionization parameter is smaller, the medium is colder, and only infrared, optical and UV lines are emitted. The column density of the ionized region emitting these lines depends on the ionization parameter, but it never reaches a Thomson thickness of unity. The continuum underlying the lines is optically thin. For instance ultraviolet lines can only be reabsorbed in the Balmer and Paschen continuum, which are optically thin because the excited levels of $\mathrm{HI}$ are not populated at low temperature. Once emitted, these line photons are therefore not reabsorbed by continuum processes (except possibly by internal dust). The situation is thus simpler than for an X-ray emitting medium. On the other hand, $\tau_{0}$ is larger in the optical range than in the X-ray range by a factor $v_{\mathrm{X}} / v_{\mathrm{opt}}$, so resonance lines are very thick in a typical BLR cloud, and escape probabilities can be used. Finally the influence of the attenuated incident continuum on line excitation is not important, except possibly for subordinate lines whose lower level is not much populated. It was indeed stressed in Collin-Souffrin \& Dumont (1986) that the use of escape probability can lead to wrong results for the computed Balmer intensities.

To summarize, the use of escape probability for the BLR is not too bad, both because the emission lines are either optically very thick (resonance lines) or very thin (forbidden lines), and because the continuum underlying these lines is optically thin.

\subsection{Our method}

In order to study X-ray emitting media, we have developed since several years a photoionization code, Titan, which does not make use of escape probability approximations, but solves the transfer both in the lines and in the continuum (cf. Dumont et al. 2000; Dumont \& Collin 2001). In its last version, Titan uses a method called "Accelerated Lambda Iteration" (ALI), which ensures that all lines are computed with an accuracy better than $1 \%$ (cf. D03 for a summary of the ALI method).

Without giving a full description of Titan, it is worthwhile mentioning that it includes the 10 most abundant elements $(\mathrm{H}$, $\mathrm{He}, \mathrm{C}, \mathrm{N}, \mathrm{O}, \mathrm{Ne}, \mathrm{Mg}, \mathrm{Si}, \mathrm{S}, \mathrm{Fe})$ and their ion species, i.e. 102 ions. H-like, He-like, Li-like ions, O IV and O V, include a multi-level description up to a quantum number $n_{\lim }: n_{\lim }=5$ for $\mathrm{H}$-like, and Li-like ions, and $n_{\mathrm{lim}}=3 \mathrm{He}$-like ions and for $\mathrm{O} I \mathrm{~V}$ and $\mathrm{O} \mathrm{V}$. The other ions are treated as two-level atoms plus a continuum (they include several resonance lines, but without interlocking). For Li-like ions, we assume mixing of the different terms of level 3, 4, and 5. In the case of He-like ions, the atom is made up of all terms for $n=2$ and $n=3$, and 2 super-levels gather the singlet and triplet levels for $n=4$. Thus the atom is represented by 15 levels plus a continuum. This better representation is chosen in order to get correct "triplet line" intensities, as they can be separated and are often used as diagnostics for the physical conditions of X-ray emitting plasma. Recombinations onto levels $n>n_{\text {lim }}$ are taken into account in a simplified way (they are either distributed on all the levels, or only on the highest one, whose population is thus overestimated). We are thus aware that from the point of view of atomic data Titan is not comparable to codes which take into account a larger number of levels for each ion species, and several thousands of transitions.

Titan solves the ionization equilibrium of all ion species of each element, the thermal equilibrium, the statistical equilibrium of all levels of each ion - all the physical processes from 
Table 1. Characteristics of the models displayed in the figures.

\begin{tabular}{|c|c|c|c|c|c|c|c|}
\hline \multicolumn{4}{|c|}{ Model } & \multicolumn{2}{|c|}{$\tau$ (cont) at $\mathrm{O}$ VIII L $\alpha$} & \multicolumn{2}{|c|}{$\overline{\tau_{0}(\mathrm{O} \text { VIII L } \alpha)}$} \\
\hline No & $\mathrm{CD}\left(\mathrm{cm}^{-2}\right)$ & $\xi$ & $V_{\text {turb }}\left(\mathrm{km} \mathrm{s}^{-1}\right)$ & ALI & escape & ALI & escape \\
\hline 1 & $10^{15}$ & 10 & 0 & $8 \times 10^{-8}$ & $8 \times 10^{-8}$ & $5.3 \times 10^{-4}$ & $5.3 \times 10^{-4}$ \\
\hline 2 & $10^{18}$ & 10 & 0 & $8.5 \times 10^{-5}$ & $8.5 \times 10^{-5}$ & 0.53 & 0.53 \\
\hline 3 & $10^{18}$ & 10 & 0 & $8.7 \times 10^{-3}$ & $8.7 \times 10^{-3}$ & 53.1 & 53.1 \\
\hline 4 & $10^{21}$ & 10 & 0 & $8.7 \times 10^{-2}$ & $8.7 \times 10^{-2}$ & 506 & 506 \\
\hline 5 & $10^{22}$ & 10 & 0 & 1.10 & 1.17 & 2760 & 2680 \\
\hline 6 & $10^{22}$ & 10 & 300 & 1.23 & 1.43 & 61 & 32 \\
\hline 7 & $10^{22}$ & 100 & 0 & $7.9 \times 10^{-2}$ & $9.4 \times 10^{-2}$ & 2241 & 1500 \\
\hline 8 & $10^{22}$ & 300 & 0 & $9.5 \times 10^{-3}$ & $1.05 \times 10^{-2}$ & 260 & 178 \\
\hline 9 & $10^{23}$ & 100 & 0 & 1.71 & 2.35 & 33180 & 19300 \\
\hline 10 & $10^{24}$ & 1000 & 0 & 0.28 & 0.36 & 5328 & 4250 \\
\hline
\end{tabular}

each level being taken into account - coupled with the transfer of the continuum and of about 900 lines. Comptonization of the continuum above $20 \mathrm{keV}$ is taken into account through the coupling with a Monte Carlo code, and comptonization of all lines is computed in an approximate way (cf. D03). Note that though it always plays a role, it does not change appreciably the whole line intensity in the cases considered here. Moreover, it is treated exactly in the same way with the escape and the transfer treatments.

Finally we should mention that presently Titan is dealing only with complete frequency redistribution. In the future we shall implement partial redistribution frequency in Titan. Here all computations have been performed assuming complete redistribution within Doppler core for the first resonance line of He- and H-like species, and within Voigt profiles for the other lines. The latter assumption does not have a strong influence on the results, since subordinate lines are optically thin or moderately thick, and the other resonance lines are not intense.

\section{Results}

\subsection{The models}

Like in D03, we have compared the spectra obtained when using Titan in its complete version, with those obtained with Titan when the line transfer is replaced by the escape probabilities in the computation of the statistical and ionization equilibrium, of the energy balance, and of the emitted line fluxes. The rest of the computations are strictly identical. This ensures that we compare exactly the same models. Indeed it would not be possible to compare directly the results obtained using another photoionization code with those of Titan, owing to the difference in the transfer of the continuum and in the atomic data.

In the following computations, we have chosen one of the escape probability approximations used in D03, Escape 14 bis, which we think to be the best for reasons explained in D03. Radiative excitation by the attenuated incident radiation was added, as it is important for optically thin media. The equations corresponding to this approximation are given in the Appendix.

Our models are plane-parallel slabs of constant hydrogen density $n_{\mathrm{H}}=10^{7} \mathrm{~cm}^{-3}$, and total hydrogen column density from $10^{15}$ to $10^{24} \mathrm{~cm}^{-2}$, irradiated on one side by an incident continuum with a spectral distribution $F_{v} \propto v^{-1}$ extending from $0.1 \mathrm{eV}$ to $100 \mathrm{keV}$. We have chosen this density, as it is probably intermediate between that of the Warm Absorber in Seyfert 1 and that of the more dilute X-ray emitting medium in Seyfert 2. Moreover, it was shown in Coupé et al. (2003) that the density has not a strong impact on the line spectrum between $10^{7}$ and $10^{12} \mathrm{~cm}^{-3}$. It is due to the fact that the most intense lines - resonance lines of $\mathrm{H}$ - and He-like ions - are formed by recombination. We call ionization parameter at the surface of the irradiated slab $\xi=4 \pi F / n_{\mathrm{H}}$, where $F$ is the integrated incident flux. We choose the value of the ionization parameter so as to get spectra dominated in the soft $\mathrm{X}$-ray range by $\mathrm{H}$-like and $\mathrm{He}$ like lines of $\mathrm{C}, \mathrm{N}$, and $\mathrm{O}$, since those are the lines observed in Seyfert 1 and 2 spectra. We were obliged to increase the value of the ionization parameter for the largest column densities, otherwise only a small fraction of the slab would be hot and $\mathrm{X}$-ray emitting, and the spectra would be quite similar to low column density cases. When the column density and the ionization parameter are large, several ion species are present in successive layers, and the spectrum contains also $\mathrm{H}-$ and $\mathrm{He}-$ like lines of more heavier species (cf. Coupé et al. 2003), so basically the most intense lines are shifted towards higher frequencies. We assumed cosmic abundances with respect to hydrogen (Allen 1973): He: 0.085; C: $3.3 \times 10^{-4}$; N: $9.1 \times 10^{-5}$; O: $6.6 \times 10^{-4}$; Ne: $8.3 \times 10^{-5} ; \mathrm{Mg}: 2.5 \times 10^{-5}$; Si: $3.3 \times 10^{-5}$; S: $1.6 \times 10^{-4}$; Fe: $3.2 \times 10^{-5}$.

In the Warm Absorber or in the emitting medium of Seyfert 2, a velocity gradient may be present, which can decrease the optical thickness of the lines. In the line transfer, it plays the role of a micro-turbulent velocity which should be added to the thermal velocity. Note that if the emitting medium is made of high velocity clouds like the BLR, the velocity gradient acts only as a macroscopic velocity which does not influence the transfer (this problem is discussed in more details in Godet et al. 2004). We have thus run a model with a turbulent velocity of $300 \mathrm{~km} \mathrm{~s}^{-1}$, close to the measured width of the lines in NGC 1068 (Kinkhabwala et al. 2002).

Table 1 summarizes the characteristics of a few models successively the column density $C D$, the ionization parameter $\xi$, the turbulent velocity $V_{\text {turb }}$, the total optical thickness of the continuum at the position of the O VIII L $\alpha$ line at $653 \mathrm{eV}$, 
and the total optical thickness at the center of the line, both for ALI and the escape computation ${ }^{2}$.

An immediate result is that the opacity of the continuum increases with the column density up to a limit of $\sim 2$. This is because the heavy elements become completely ionized for large values of the ionization parameter, which are required to create a thick hot layer. The same phenomenon exists for $\tau_{0}$ : it cannot exceed a value corresponding to the maximum column density of the ion species, reached for an optimum value of the ionization parameter (cf. Coupé et al. 2003 for more detailed explanations). Model 7 with a column density of $10^{22} \mathrm{~cm}^{-2}$ and $\xi=100$ has a continuum opacity 20 times smaller than for $\xi=10$, but it has the same value of $\tau_{0}(\mathrm{O}$ VIII L $\alpha)$, because $\mathrm{O}$ VIII is still the dominant ion, while $\mathrm{C}$ and $\mathrm{N}$ are already completely ionized. On the contrary, Model 8 with the same column density but $\xi=300$ has a smaller value of $\tau_{0}(\mathrm{O}$ VIII $\mathrm{L} \alpha$ ), because O VIII is no more the dominant ion, it is replaced by O IX. One sees the strong decrease of the line optical thickness in the presence of a large micro-turbulent velocity, while the continuum opacity is almost unchanged. Note also that the continuum opacity and $\tau_{0}(\mathrm{O}$ VIII $\mathrm{L} \alpha)$ vary in opposite directions: the first is smaller for the transfer treatment, and the second for the escape approximation. It is linked to the slight variation of the ionization state in the two treatments.

\subsection{The case of Seyfert 2: The reflected spectrum}

We try first to mimic the conditions of the X-ray emitting regions of Seyfert 2 galaxies. According to the Unified Scheme of Seyfert galaxies, we are observing in the X-ray range the "reflection" of the central source produced by a photoionized "mirror", and we do not see the primary photoionizing continuum. It is actually not a purely reflecting medium, as it reprocesses the radiation (however we shall call this emission the "reflected" spectrum). This region has probably a conical geometry, and we should observe almost perpendicularly to the cone axis. Of course our representation of such a situation can be only very crude, as Titan is dealing with a plane-parallel and not a spherical or conical geometry.

Since lines having the largest equivalent widths $(E W s)$ are best observed, and since very often $E W \mathrm{~s}$ are given in the literature, we display the results as $E W \mathrm{~s}$ with respect to the reflected continuum instead of line fluxes. Note that the spectrum "reflected" by the illuminated side is similar to that emitted by the back side only in the case of very thin slabs, but it is different for moderately thick slabs.

Figure 1 displays the $E W$ s of the lines in the reflected spectrum, for the chosen models and for the transfer and the escape treatments. A few intense lines are identified: the spectra are dominated by $\mathrm{L} \alpha$ lines of $\mathrm{H}$-like and He-like lines ${ }^{3}$. For larger

\footnotetext{
${ }^{2}$ Since Titan is made for Thomson-thick media, the transfer is treated in the semi-isotropic two-stream Eddington approximation. It means that the optical thickness is multiplied by a factor $\sqrt{3}$ with respect to the normal direction.

${ }^{3}$ In He-like ions, the first resonant line $\left(1 \mathrm{~s}^{2}{ }^{1} \mathrm{~S}-2 \mathrm{p}^{1} \mathrm{P}^{\mathrm{o}}\right)$ is called $w$, the forbidden line $1 \mathrm{~s}^{2}{ }^{1} \mathrm{~S}-2 \mathrm{~s}^{3} \mathrm{~S}$ is called $z$, and the intercombination lines $1 \mathrm{~s}^{2}{ }^{1} \mathrm{~S}-2 \mathrm{p}^{3} \mathrm{P}_{1,2}^{\mathrm{o}}$ are called $x$ and $y$.
}

values of the ionization parameter, heavier $\mathrm{H}$ - and He-like ions are present.

The transfer and the escape treatments give different results already at very low column densities: for Model $3\left(10^{20} \mathrm{~cm}^{-2}\right)$, the error is $30 \%$ for a few lines. It is clearly not due to the influence of the continuum opacity, which is negligible, but to the interlocking between levels (and perhaps also partly to the approximate treatment of the incident continuum, as $\tau_{0}$ is not negligible). A noticeable result is that, except for very low values of the column density, the escape treatment leads to a systematic overestimation of the resonance line intensities, as already noticed in D03. It is particularly obvious for $\mathrm{L} \alpha$ of $\mathrm{H}$ like ions, and in a lesser amount for the w-term of He-like ions. The overestimation is smaller in the case of forbidden or subordinate lines.

Figures 2 and 3 can help to understand these discrepancies. Figure 2 displays the ratios of the upper to lower populations $N_{\text {up }} / N_{\text {low }}$, as functions of the position in the slab, for several transitions of O VIII and O VII, for Model 5 (column density $10^{22} \mathrm{~cm}^{-2}, \xi=10$, no turbulent velocity). This ratio is proportional to the source function of the lines. The figure shows that for the two resonance lines, O VIII L $\alpha$ and O VII w, it differs strongly in the whole slab with the two treatments, contrary to those of the other transitions. Figure 3 displays the NRB (for the transfer) and the escape probability $P_{\text {esc }}$ (for the escape treatment), as functions of the position in the slab, for these two lines. $P_{\text {esc }}$ is the sum of the escape probabilities towards the illuminated and towards the back side (cf. the Appendix), which are also shown on the figure. In principle it should be equal to the NRB, but we see that they have almost nothing in common. The reflected flux is mainly provided by the region located at $10^{13} \leq z \leq 10^{14} \mathrm{~cm}$ from the surface, where the source functions are overestimated by more than one order of magnitude with the escape treatment.

To also help understanding the difference with a "cold" medium like the BLR, we show for the same model the fractional abundances of several ions able to efficiently absorb the O VIII L $\alpha$ photons on the top panel of Fig. 4. We see that they are dominant through the whole slab. The bottom panel displays the emissivity of the O VIII L $\alpha$ line in the escape computation, as a function of the depth, and the optical thickness in the continuum at the position of the line, starting from the illuminated side. The emissivity is important up to $z=5 \times 10^{14} \mathrm{~cm}$, where the optical thickness reaches a value of the order of 0.5 . Thus the reflected line is attenuated by about a factor two. However it is not sufficient to explain the discrepancy between the escape and the transfer treatments, and one should also invoke the other reasons mentioned previously.

The errors on the escape results increase with the column density and reach a factor five for Model $9\left(10^{23} \mathrm{~cm}^{-2}, \xi=\right.$ $100)$, where both the line and the continuum opacities are large, so the influence of the diffuse continuum becomes important. They decrease for Model $10\left(10^{24} \mathrm{~cm}^{-2}, \xi=1000\right)$, because the opacities of the continuum and the lines are smaller. Note also that the errors are quite small with Model $8\left(10^{22} \mathrm{~cm}^{-2}, \xi=\right.$ $300)$. It is easily understood: this model is highly ionized, and the column density is not large enough to allow the presence of low ionized species. As a result, the spectrum is mostly made 

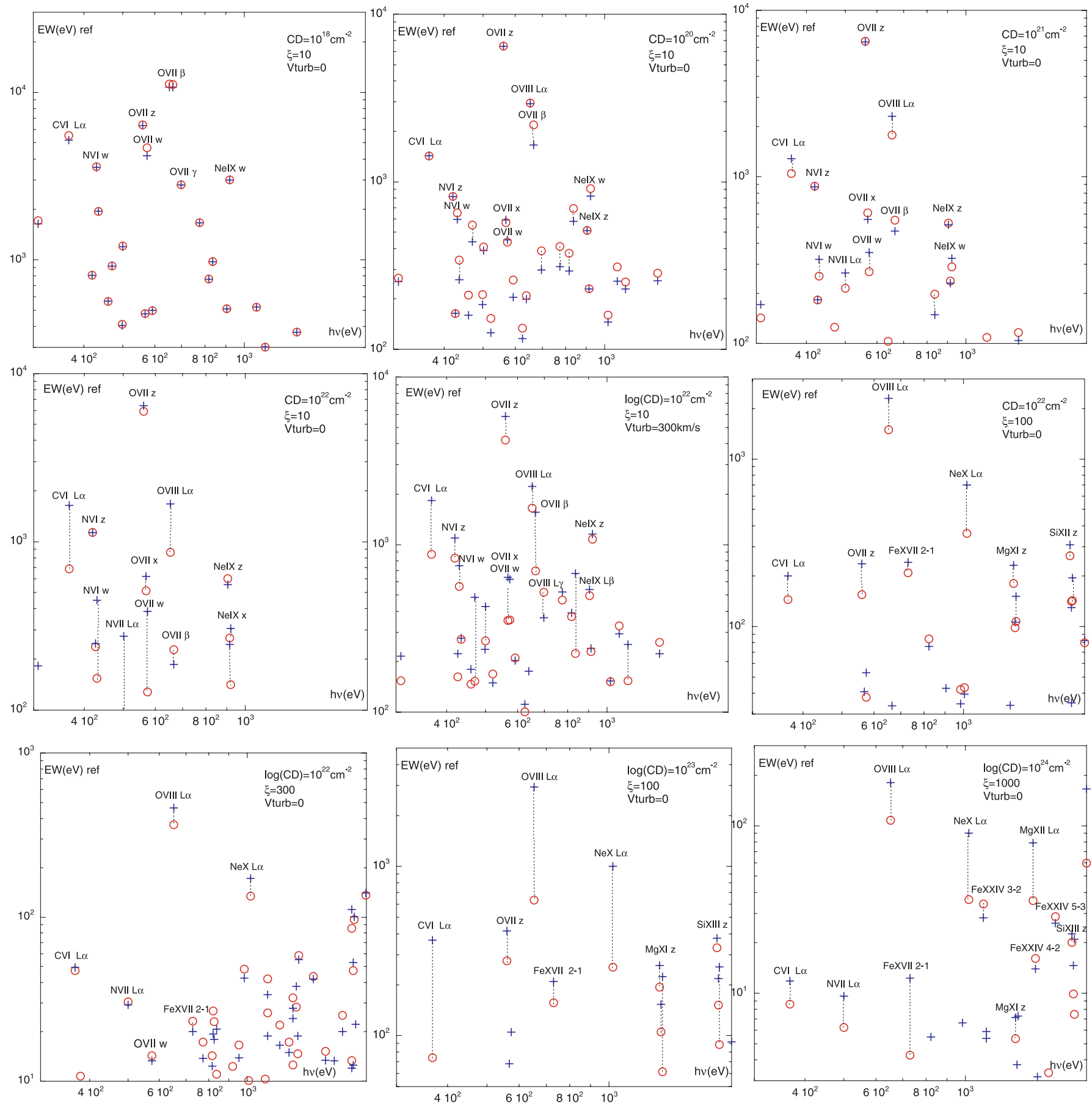

Fig. 1. $E W \mathrm{~s}$ (in $\mathrm{eV}$ ) of the lines in the reflected spectrum, for the different models, with the transfer (red circles) and with the escape treatment (blue crosses).

of He- and H-like lines of heavy elements and of O VIII, and it does not display the O VII lines characteristic of a Seyfert 2 spectrum, as can be seen in Fig. 1.

The comparison of Models 5 and $6\left(10^{22} \mathrm{~cm}^{-2}, \xi=10\right.$, respectively without and with a micro-turbulent velocity of $300 \mathrm{~km} \mathrm{~s}^{-1}$ ) shows that the escape probability approximation gives slightly better results in the presence of a micro-turbulent velocity, as expected since the lines are less optically thick. Still the errors on line intensities and line ratios are typically of the order of a factor two. Moreover, the results are shifted towards smaller column densities for a given $E W$ (Godet et al. 2004): here, for a turbulent velocity of $300 \mathrm{~km} \mathrm{~s}^{-1}$, the $E W \mathrm{~s}$ of the resonance lines are similar to those without turbulent velocity, but for a column density about one order of magnitude smaller. The comparison of Model 5 with Model $7\left(10^{22} \mathrm{~cm}^{-2}, \xi=100\right)$ shows also that the escape probability approximation gives a better result in the latter case, because the continuum opacity is small. Also, due to the higher ionization parameter, the spectrum displays more intense lines of He- and H-like heavy elements. 

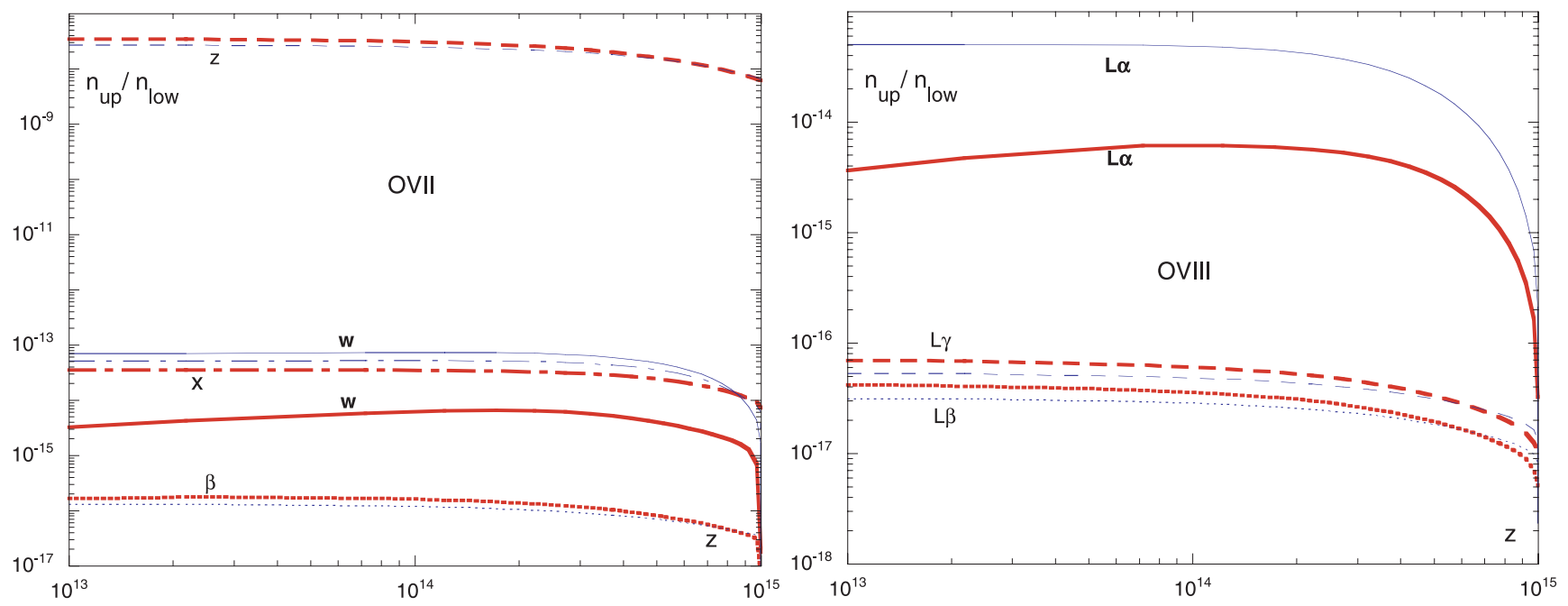

Fig. 2. Ratios $n_{\text {up }} / n_{\text {low }}$ as functions of the position in the slab, for several transitions of O VIII and O VII, for Model 5 (column density $10^{22} \mathrm{~cm}^{-2}$, $\xi=10$, no turbulent velocity): thick red lines: transfer, thin blue lines: escape.
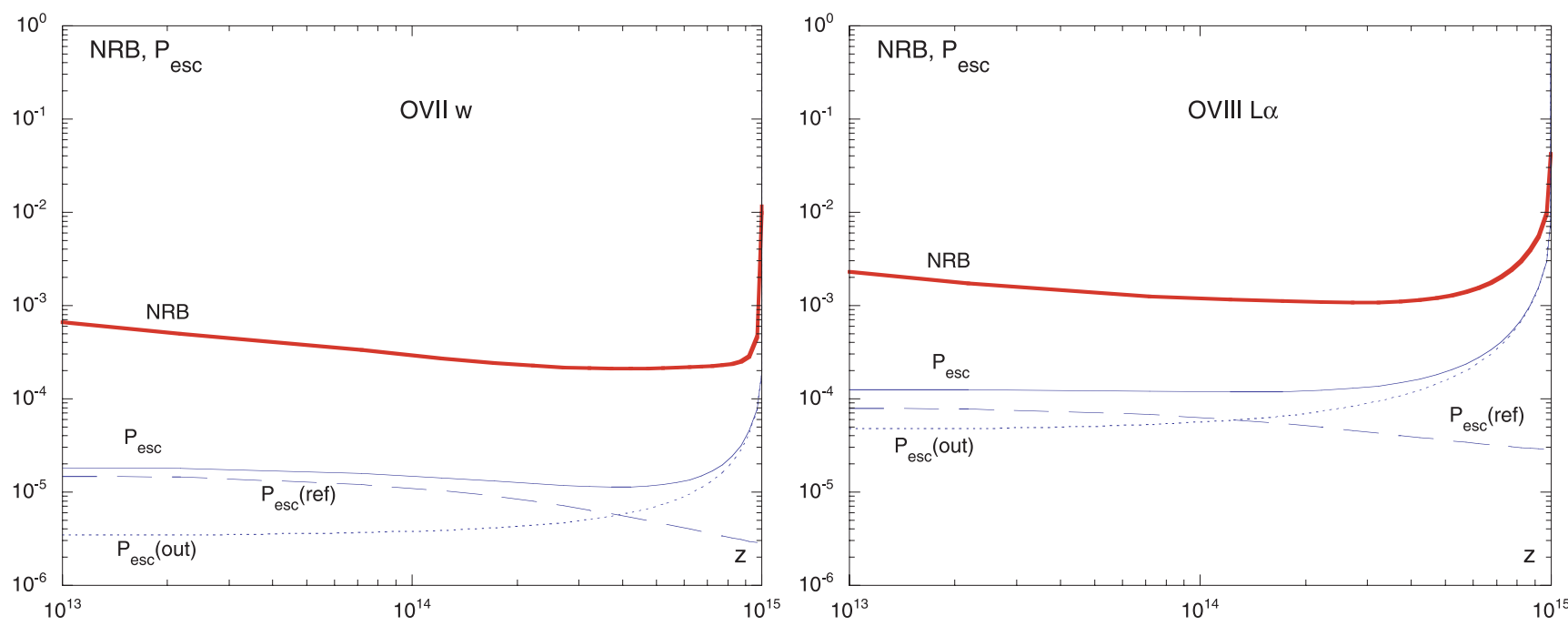

Fig. 3. NRB (for the transfer) and escape probability $P_{\text {esc }}$ (for the escape treatment), as functions of the position in the slab, for O VIII L $\alpha$ and O VII w, for Model 5 (column density $10^{22} \mathrm{~cm}^{-2}, \xi=10$, no turbulent velocity). The figure shows also the escape probabilities towards the illuminated and towards the back side, respt. $P_{\text {esc }}$ (ref) and $P_{\text {esc }}$ (out) (see the text for explanations). Note that $P_{\text {esc }}\left(\right.$ ref) and $P_{\text {esc }}($ out) are much smaller than their values at the surface, 0.5 .

In summary, the errors in the line intensities and line ratios due to the use of the escape approximation are not easy to predict, as they depend on different processes in a complex and subtle way. They are important even for low column densities.

To illustrate more clearly the effects of the use of escape probabilities on the observed spectrum, Fig. 5 displays the reflected spectrum for two models, in the same form as they are generally published in the literature. Though the column densities are relatively small $\left(10^{22}\right.$ and $\left.10^{23} \mathrm{~cm}^{-2}\right)$, large discrepancies appear between the escape and the transfer treatments, which can lead to misinterpretations of the observed spectra when modeled by the escape treatment.

The ratio $G=(z+x+y) / w$ of He-like ions is used as a diagnostic for hot plasma. The variation of this ratio as a function of several parameters is studied in Coupé et al. (2003) and Godet et al. (2004). Here we focus only on the differences between the escape and the transfer treatment. Figure 6 displays this ratio versus the column density for the models studied here. Again we see that the differences are the largest (a factor three) for Models 5 and 8. It is however interesting to notice that in the presence of a turbulent velocity, $G$ is almost the same for both treatments.

\subsection{The case of Seyfert 1: The emitted spectrum}

In Seyfert 1, one observes directly the central X-ray continuum, possibly after absorption by the Warm Absorber. About 50\% of Seyfert galaxies have absorption lines, and it is most probable that the "Warm Absorber" is present in all active nuclei, but it does not cross always the line of sight of the X-ray continuum. Emission lines are produced by the Warm Absorber, but they are diluted by the presence of the primary continuum. The observed spectrum is thus the sum of reflection from the illuminated side and emission from the back side of the 


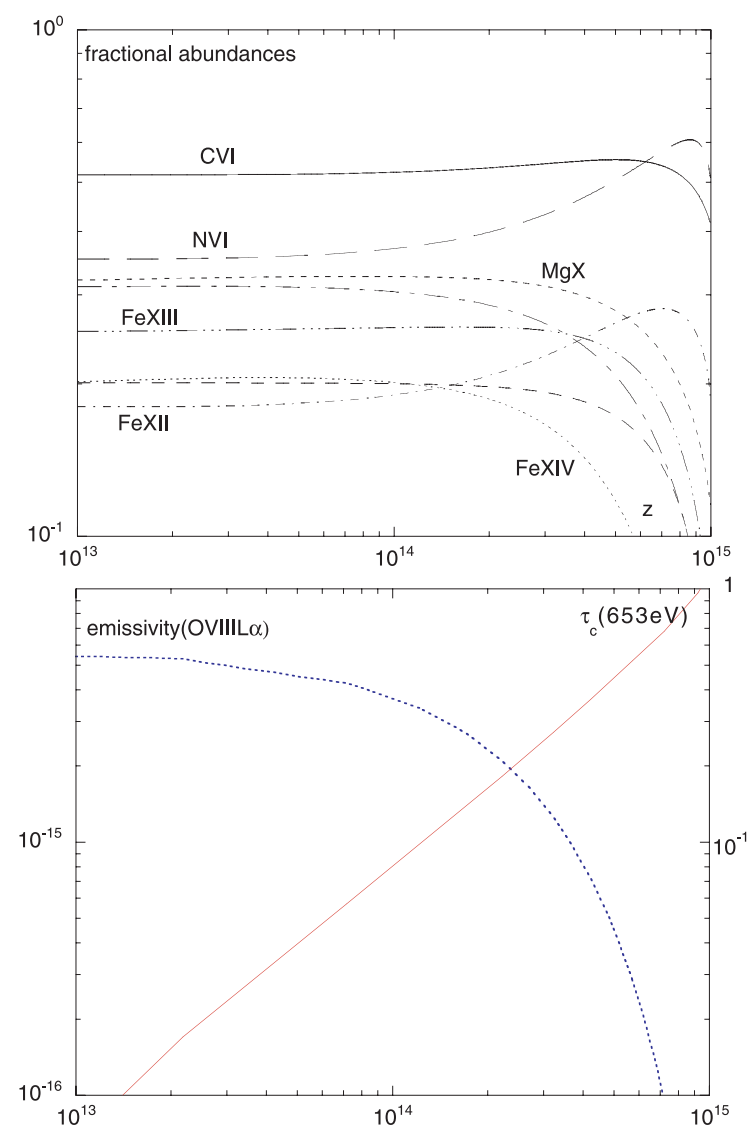

Fig. 4. Fractional abundances of several ions able to absorb the O VIII $\mathrm{L} \alpha$ photons (top panel), and emissivity of the O VIII L $\alpha$ line and optical thickness in the continuum at the position of the line (bottom panel), as functions of the position in the slab, for Model 5 (column density $10^{22} \mathrm{~cm}^{-2}, \xi=10$, no turbulent velocity).
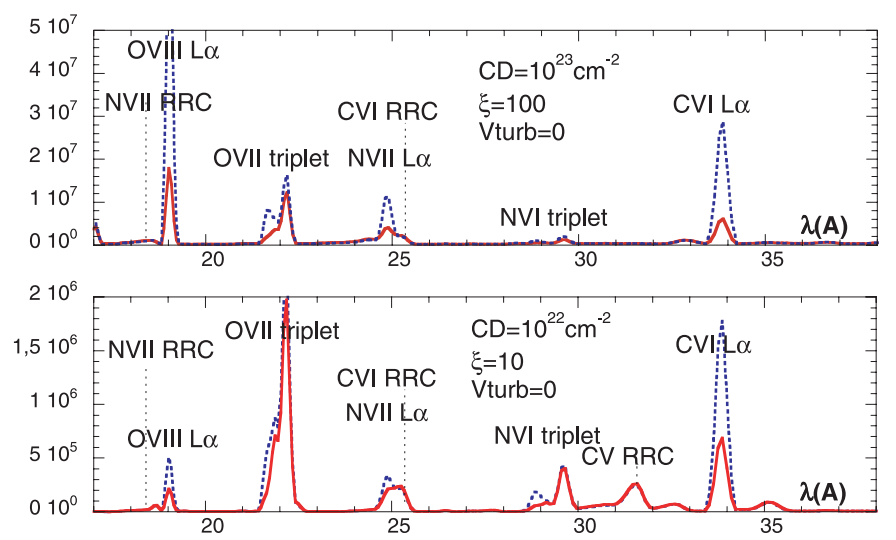

Fig. 5. Reflected spectrum for two models: solid red line: transfer; dashed blue line: escape probability. The ordinate is proportional to $F_{\lambda}$ in photon number (actually it is equal to $v F_{v}$ ). The spectra are displayed with a spectral resolution of $R=30$.

clouds, plus the primary continuum, absorbed if the line of sight crosses the Warm Absorber. If it is distributed spherically or axi-symmetrically, the proportions coming from each side

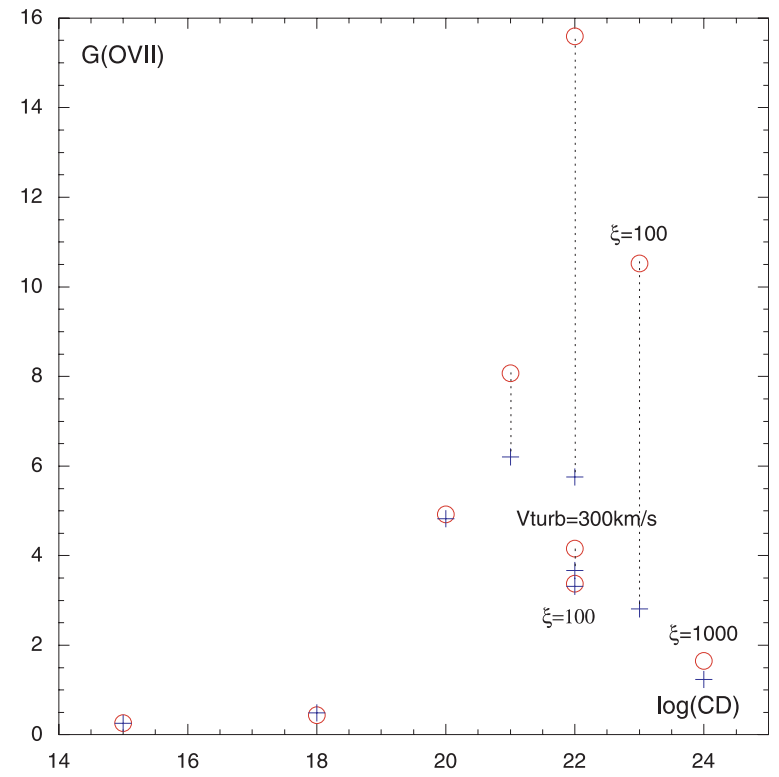

Fig. 6. G ratios (see the text) in the reflected spectrum versus the column density, for the transfer (red circles) and the escape treatment (blue crosses). When another value is not indicated, $\xi=10$.

are identical. Assuming a coverage factor $f$, the observed $E W \mathrm{~s}$ are thus:

$E W=\frac{f(1-f) E W(\text { ref }) F_{v}(c, \text { ref })+f E W(\text { out }) F_{v}(c, \text { out })}{f(1-f) F_{v}(c, \text { ref })+f F_{v}(c, \text { out })+F_{v}(c, \text { prim })}$

where $F_{v}(c$, ref $), F_{v}(c$, out $), F_{v}(c$, prim $)$, are the continuum flux, respectively reflected, emitted outward, and the primary continuum (absorbed or not), at the line frequency. If the line of sight crosses the Warm Absorber, there are also absorption lines imprinted on the primary continuum. We will assume that they are not located at the same frequencies as the emission lines, owing to the existence of velocity gradients (for instance in the case of a wind, the emission lines are redshifted with respect to the absorption lines).

As an illustration, Fig. 7 displays the $E W$ s of the emission lines produced by the Warm Absorber with a covering factor $f=0.5$ : 1 - not crossing the line of sight (top panels), 2 - crossing the line of sight (bottom panels). Comparing these results to those of Seyfert 2, we see that the emission line spectra are very similar in both cases. However when the primary continuum is not absorbed (top panel), the $E W$ s are always very small, and only a few lines could be detectable (O VIIz, O VIII L $\alpha$, C VI L $\alpha$ ). When the line of sight crosses the Warm Absorber, the $E W \mathrm{~s}$ are larger, and many lines should be detected for a column density of the order of $10^{22} \mathrm{~cm}^{-2}$ or larger. So it is a powerful way to estimate the column density of the Warm Absorber, independent of the absorption lines. Note also that the line ratios differ between the case with and without absorption of the primary continuum, owing to the variation of the continuum opacity with the frequency.

Anyway, our purpose in this paper is not to study the emissive regions of Seyfert nuclei, but to estimate the validity of the computations performed with the escape approximation. Figure 7 shows that, like in the case of Seyfert 2, the EWs are overestimated by large factors (up to 5 for Model 9) by 

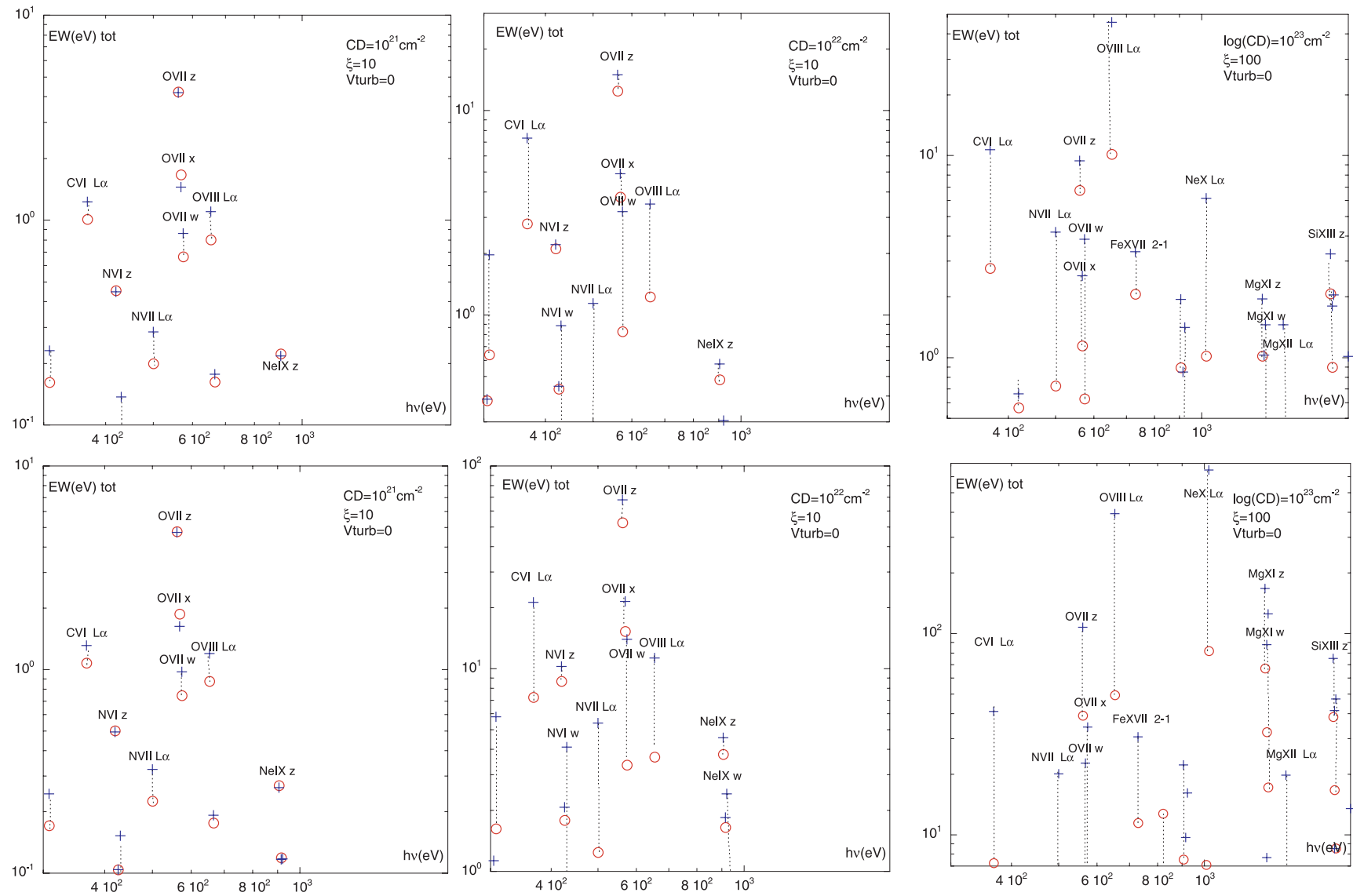

Fig. 7. EWs (in eV) of the lines, in the case of a spherical "Warm Absorber" with a coverage factor of 0.5 , for different models computed with the transfer (red circles) and with the escape treatment (blue crosses). The top panels correspond to the line of sight of the central X-ray source not crossing the Warm Absorber, and the bottom panels to the Warm Absorber crossing the line of sight (cf. the text).

the approximate treatment. Again also, the overestimation is much larger for resonance lines than for forbidden or subordinate lines.

\section{Conclusion}

We have shown that the use of the escape probability approximation leads to large errors in the computed line fluxes and line ratios, in conditions which are typical of the X-ray emitting regions of Seyfert 2 nuclei, and of the Warm Absorber of Seyfert 1, i.e. for a Thomson thickness of the order of 0.001 or larger. This completes the previous paper (D03), where the same study was performed for thicker media, and where it was shown that the use of the escape approximation leads to errors by more than one order of magnitude on the line fluxes and line ratios. We find here that the errors are of the order of $30 \%$ for a column density of $10^{20} \mathrm{~cm}^{-2}$, and can reach a factor five for a column density $10^{23} \mathrm{~cm}^{-2}$. We confirm that they are almost always in the direction of an overestimation of the most intense line intensities, especially of the $\mathrm{L} \alpha$ line of $\mathrm{H}$-like ions, and of the resonance w-term of He-like ions. We explain why such large errors occur for X-ray emitting media, and not for cooler media like the BLR.

The comparison between the escape approximation and the transfer treatment (performed through the Accelerated Lambda Iteration method with our photoionization code Titan), is made in such a way that no other possible explanation of the discrepancies than the use of the escape probability approximation can be invoked. So one is led to conclude that unless a real transfer of the lines is introduced in the codes for modelling X-ray spectra, the results cannot have an accuracy better than that given by the approximation, even when the treatment of atomic physics is highly sophisticated.

Whatever the discrepancy between the results of the escape probability and the transfer treatment, an uncertainty remains concerning the real intensity of the resonance lines. Here they were computed assuming complete redistribution within Doppler core, which mimics partial redistribution within a Voigt profile. PRD cannot be taken into account accurately with the escape probability formalism, but it can be done with the transfer treatment, provided the implementation in the code of another substantial time consuming procedure. We are presently studying such an improvement.

\section{Appendix: Equations used for the escape approximation in the optically thin case}

We compute the escape probability towards the surface $P_{\mathrm{e}}\left(\tau_{0}\right)$ of a line as (cf. Collin-Souffrin et al. 1981):

$P_{\mathrm{e}}\left(\tau_{0}\right)=\max (f 1, f 2)$, 
for all subordinate and high resonance lines, and

$P_{\mathrm{e}}\left(\tau_{0}\right)=f 1$

for the first resonance lines of $\mathrm{H}$ - and He-like species, with

$f 1=\frac{0.5}{1+2 \tau_{0} \sqrt{\pi \ln \left(\tau_{0}+1\right)}} \quad f 2=\frac{2}{3} \sqrt{\frac{a}{\sqrt{\pi} \tau_{0}}}$,

where $\tau_{0}$ is the optical thickness at the line center between the emission point and the surface (we recall that it is taken along the photon path, so it is multiplied by a factor $\sqrt{3}$ to take into account the fact that the direction of the emitted photon is at random, and to be able to compare the escape approximation to the transfer treatment), and $a$ is the usual damping constant. $f 1$ corresponds to the Doppler core, and $f 2$ to the Lorentz wings of the Voigt profile.

The total escape probability $P_{\text {esc }}$ is the sum of the escape probability towards the illuminated side, $\beta_{\text {ref }}=P_{\mathrm{e}}\left(\tau_{0}\right)$, and towards the back side, $\beta_{\text {out }}=P_{\mathrm{e}}\left(T_{0}-\tau_{0}\right)$, where $T_{0}$ is the total optical thickness of the slab depth at the line center.

In the equations for the level populations, the net radiative rate from an excited level is replaced by $A_{\mathrm{ul}} \beta_{\mathrm{pop}}$, with

$\beta_{\text {pop }}=\min \left[1, P_{\mathrm{esc}}(\tau) \times\left(1+\frac{\kappa_{\mathrm{c}}}{\kappa_{\mathrm{l}}} F\left(\frac{\kappa_{\mathrm{c}}}{\sqrt{\kappa_{\mathrm{l}}}}\right)\right)\right]$,

where $\kappa_{\mathrm{c}}$ and $\kappa_{1}$ are respectively the absorption coefficient in the continuum and in the line, and $F$ is the operator given by Hummer (1968) to account for destruction by continuum absorption in one line scattering:

$F(X)=\int_{-\infty}^{\infty} \frac{\phi(x)}{X+\phi(x)} \mathrm{d} x$

where $\phi(x)$ is the absorption line profile, $x=\delta v / \delta v_{\mathrm{D}}$, and $\delta v_{\mathrm{D}}$ is the Doppler width.

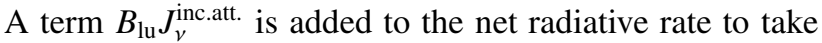
into account excitations by the attenuated incident radiation $J_{v}^{\text {inc.att. }}=2 \beta_{\text {ref }} /(2 \pi) F_{v}^{\text {inc }}$, according to the definition of the flux, $F_{v}^{\text {inc }}$ being the incident flux at the frequency $v$ (the corresponding deexcitation rate is negligible).

The local cooling for each line is:

$\Lambda_{\text {line }}=\left(n_{\mathrm{u}} A_{\mathrm{ul}} P_{\mathrm{esc}}-n_{\mathrm{l}} B_{\mathrm{lu}} J_{v}^{\text {inc.att. }}\right) \frac{h v}{n_{\mathrm{e}} n_{\mathrm{H}}}$.

The reflected flux in a line is computed as:

$F_{\text {ref }}=\int n_{\mathrm{u}} A_{\mathrm{ul}} h v \beta_{\text {ref }} \exp \left[-\tau_{\mathrm{e}}\right] \mathrm{d} z$

where $\tau_{\mathrm{e}}$ is the effective optical thickness of the slab in the continuum at the line frequency, between the current point and the illuminated surface.

In the outward emitted line flux, one must take into account the photons absorbed in the incident continuum:

$F_{\text {out }}=\int\left(n_{\mathrm{u}} A_{\mathrm{ul}} h v \beta_{\text {out }} \exp \left[-\left(T_{\mathrm{e}}-\tau_{\mathrm{e}}\right)\right]-n_{\mathrm{l}} B_{\mathrm{lu}} J_{v}^{\text {inc.att. }}\right) \mathrm{d} z$

where $T_{\mathrm{e}}$ is the effective total optical thickness of the slab in the continuum at the line frequency.
The ionization rate due to the lines, at the depth $z$, is equal to:

$$
\begin{gathered}
\sqrt{3} \kappa_{\mathrm{c}} A_{\mathrm{ul}}\left(\int_{0}^{z}\left(n_{\mathrm{u}} \beta_{\mathrm{out}} \exp \left[-\tau_{\mathrm{e}}+T_{\mathrm{e}}\right]-n_{\mathrm{l}} \frac{B_{\mathrm{lu}}}{A_{\mathrm{ul}}} J_{v}^{\text {inc.att. }}\right) \mathrm{d} Z\right. \\
\left.+\int_{H}^{z} n_{\mathrm{u}} \beta_{\mathrm{ref}} \exp \left[-T_{\mathrm{e}}+\tau_{\mathrm{e}}\right] \mathrm{d} Z\right)
\end{gathered}
$$

This expression is used also for the gains by photoionizations due to the lines.

The escape probability is slightly different in the case of a highly ionized and/or moderately thick medium, as one must take into account the shift of line photons by comptonization. Thus $\beta_{\text {ref }}$ is replaced by $\beta_{\text {ref }}^{\prime}=\beta_{\text {ref }}+\frac{1}{2}\left(1-P_{\text {esc }}(\tau)\right) \frac{\sigma}{\kappa_{1} \sqrt{\pi}+\kappa_{\mathrm{c}}+\sigma}$, and $\beta_{\text {out }}$ by $\beta_{\text {out }}^{\prime}=\beta_{\text {out }}+\frac{1}{2}\left(1-P_{\text {esc }}(\tau)\right) \frac{\sigma}{\kappa_{1} \sqrt{\pi}+\kappa_{\mathrm{c}}+\sigma}$, and $P_{\mathrm{e}}$ is replaced by $P_{\mathrm{e}}^{\prime}=\beta_{\text {ref }}^{\prime}+\beta_{\text {out }}^{\prime}$.

\section{References}

Allen, C. W. 1973, Astrophysical quantities, University of London (The Athlone Press)

Antonucci, R. R. J., \& Miller, J. S. 1985, ApJ, 297, 621

Athay, G. 1972, Radiation Transport in Spectral Lines (D. Reidel Publishing Company)

Avrett, E. H., \& Loeser, R. 1987, Num. Rad. Trans., 135, ed. Kalkoven, CUP

Collin-Souffrin, S., Delache, P., Frisch, H., \& Dumont, S. 1981, A\&A, 104, 264

Collin-Souffrin, S., \& Dumont, S. 1986, A\&A, 166, 13

Coupé, S., Godet, O., Dumont, A.-M., \& Collin, S. 2003, A\&A, in press

Dumont, A.-M., Abrassart, A., \& Collin, S. 2000, A\&A, 357, 823

Dumont, A., \& Collin, S. 2001, Spectroscopic Challenges of Photoionized Plasmas, ASP Conf. Ser., 247, 231

Dumont, A.-M., Collin, S., Paletou, F., et al. 2003, A\&A, 407, 13 (D03)

Elitzur, M. 1982, Rev. Mod. Phys., 54, 1125

Ferland, G. J., Korista, T., Verner, D. A., et al. 1998, PASP, 110, 761

Godet, O., Collin, S., \& Dumont, A.-M. 2004, A\&A, submitted

Hubeny, I. 2001, Spectroscopic Challenges of Photoionized Plasmas, ASP Conf. Ser., 247, 197

Hummer, D. G. 1968, MNRAS, 138, 73

Kalkofen, W. 1984, Methods in radiative transfer (Cambridge University Press)

Kalkofen, W. 1987, Numerical Radiative Transfer (Cambridge University Press)

Kallman, T. R., \& Krolik, J. H. 1995, XSTAR, a Spectral Analysis Tool, Users Guide

Kinkhabwala, A., Sako, M., Behar, E., et al. 2002, ApJ, 575, 732

Milkey, R. W., \& Mihalas, D. 1973, ApJ, 185, 709

Netzer, H. 1975, MNRAS, 171, 395

Netzer, H. 1993, ApJ, 411, 594

Ogle, P. M., Brookings, T., Canizares, C. R., Lee, J. C., \& Marshall, H. L. 2003, A\&A, 402, 849

Sako, M., Kahn, S. M., Paerels, F., \& Liedahl, D. A. 2000, ApJ, 543, L115

Vernazza, J. E., Avrett, E. H., \& Loeser, R. 1981, ApJS, 45, 635 